\title{
Eosinophil-derived neurotoxin: a novel biomarker for diagnosis and monitoring of asthma
}

\author{
Chang-Keun Kim, MD \\ Department of Pediatrics, Asthma \& Allergy Center, Inje University Sanggye Paik Hospital, Seoul, Korea
}

Asthma is associated with increased levels of eosinophils in tissues, body fluids, and bone marrow. Elevated levels of eosinophil-derived neurotoxin (EDN) and eosinophil cationic protein (ECP) have been noted in asthma patients. Higher levels of EDN and ECP are also associated with exacerbated asthmatic conditions. Thus, EDN, along with ECP, may aid the diagnosis and monitoring of asthma. Several groups have suggested that EDN is more useful than ECP in evaluating disease severity. This may partially be because of the recoverability of EDN (not sticky, 100\% recovery rate), as ECP is a sticky and more highly charged protein. In terms of clinical utility, EDN level is a more accurate biomarker than ECP when analyzing the underlying pathophysiology of asthma. As a monitoring tool, EDN has shown good results in children with asthma as well as other allergic diseases. In children too young to fully participate in lung function tests, EDN levels may be useful as an alternative measurement of eosinophilic inflammation. EDN can also be used in adult patients and in multiple specimen types (e.g., serum, sputum, bronchoalveolar lavage fluid, and nasal lavage fluid). These results are repeatable and reproducible. In conclusion, EDN may be a novel biomarker for the diagnosis, treatment, and monitoring of asthma/allergic disease.

Key words: Eosinophil-derived neurotoxin, Biological markers, Diagnosis, Monitoring of asthma, Child

\section{Introduction}

Eosinophil-derived neurotoxin (EDN), an eosinophil degranulation product, is currently drawing much attention as a novel biomarker for the diagnosis and monitoring of asthma in both children and adults. A brief introduction to eosinophilic inflammation and eosinophil degranulation proteins will result in several clinical studies, including EDN's role in postbronchiolitis wheezing, childhood asthma, childhood allergic rhinitis (AR), chronic cough and asthma in adults, and EDN monitoring in individual clinical cases.

\section{Airway diseases and eosinophilic inflammation}

Regardless of the asthma phenotype, when eosinophils are activated (by infection, allergy, or both), they release EDN ${ }^{11}$. Thus, EDN levels can be used in the diagnosis and monitoring of different asthma phenotypes (e.g., asthma, viral wheezing, eosinophilic bronchitis, chronic cough, etc.).

Eosinophils are major players in allergic diseases ${ }^{1)}$. Therefore, the direct measurement of eosinophilic inflammation is required to diagnose, treat, and monitor patients with
Corresponding author: Chang-Keun Kim, MD Department of Pediatrics, Asthma \& Allergy Center, Inje University Sanggye Paik Hospital, 1342 Dongil-ro, Nowon-gu, Seoul 139-707, Korea Tel: +82-2-950-8832

Fax: +82-2-950-1662

E-mail: kimck@paik.ac.kr

Received: 17 April, 2012

Accepted: 9 August, 2012

Copyright (C) 2013 by The Korean Pediatric Society

This is an open-access article distributed under the terms of the Creative Commons Attribution NonCommercial License (http://creativecommons.org/ licenses/by-nc/3.0// which permits unrestricted noncommercial use, distribution, and reproduction in any medium, provided the original work is properly cited. 
asthma. However, management decisions have traditionally been based on symptoms (nonspecific and subjective), airway function, and rescue medication use $\mathrm{e}^{2,3)}$.

Current measures of airway inflammation (e.g., airway function) do not correlate well with underlying eosinophilic inflammation ${ }^{4}$. Therefore, clinicians cannot always predict the nature and extent of lower airway inflammation ${ }^{5}$. For example, eosinophilia develops weeks before exacerbation ${ }^{6,7)}$. Diagnostic assays should clearly reflect the underlying pathophysiology, but many do not. Another challenge that should be overcome is the fact that some children are too young to cooperate with lung function tests.

Currently, eosinophilic inflammation monitoring involves eosinophil cell counts/percentages that provide only a limited understanding of the activity of these cells. The secretory activity of eosinophils equals the concentrations of eosinophils times their propensity to release mediators (e.g., EDN and eosinophil cationic protein $[\mathrm{ECP}])^{8}$. One way in which these act as major effector cells is through the release of granule proteins, which induce tissue damage and dysfunction, as well as the further propagation of airway inflammation. Elevated levels of ECP and EDN have been noted in asthmatics, with higher levels of ECP and EDN found during asthma exacerbation when compared with healthy patients and those with stable asthma (SA). This would suggest that airway inflammation associated with asthma exacerbation is characterized by both an increase in the number of eosinophils and an increase in airway eosinophil degranulation.

\section{Eosinophil degranulation proteins}

Eosinophil granule proteins (EDN, ECP, major basic protein [MBP], and eosinophil peroxidase [EPO]) (Fig. 1) are the proteins implicated most strongly in asthma pathophysiology ${ }^{9)}$. MBP is found in the core, while EDN, ECP, and EPO are found in the matrix. EDN and ECP are released almost exclusively by eosinophils ${ }^{11}$. Therefore, any change in EDN or ECP levels would directly reflect changes in eosinophilic inflammation. As a monitoring tool, ECP has produced mixed results ${ }^{10-13)}$. EDN shares biochemical, antiviral, and neurotoxic properties with ECP. However, unlike ECP, EDN has little or no cytotoxicity but is more easily recoverable because it is less highly charged ${ }^{14)}$. It does stick slightly to the measurement wall or other cell surfaces. However, ECP is highly charged; therefore, the result is dependable on the time interval between when the blood is drawn and when it is measured. Despite this, ECP remains the eosinophil degranulation protein used most widely in clinical practice ${ }^{15}$. As a monitoring tool, EDN has had good results $^{10,11,16,17)}$. In Taniuchi et al.'s study ${ }^{11)}$ involving atopic dermatitis patients, the serum EDN levels in mild, moderate, and severe groups were significantly higher than that in the control group ( $P<0.01, P<0.05$, and $P<0.05$, respectively).

ECP levels in the mild group were significantly higher than in the control group $(P<0.01)$. Furthermore, serum EDN levels had the highest correlation with skin scores. In terms of clinical utility, EDN levels are a more accurate biomarker of the underlying pathophysiology of asthma (i.e., eosinophilic inflammation); consequently, they provide an objective measure of eosinophil secretory activity. The authors suggested that serum EDN is more stable than serum ECP and more strongly reflects eosinophil degranulation in vivo and in vitro.

\section{Clinical studies using EDN}

\section{Postbronchiolitis wheezing, a precursor to childhood asthma development (ELBIS study)}

Most infants recover from acute bronchiolitis, but some develop recurrent wheezing. In respiratory syncytial virus (RSV) bronchiolitis, there is evidence of eosinophil activation. During this activation, granular proteins (ECP and EDN), leukotrienes, and cytokines are released ${ }^{18)}$.

The primary objective of the early intervention with leukotriene receptor antagonist (LTRA) for RSV bronchiolitis in Seoul (ELBIS) study was to investigate the effect of montelukast, a LTRA, on eosinophil degranulation in postbronchiolitic wheezing, while the secondary objective was to determine if montelukast treatment was associated with a decrease in recurrent wheezing postbronchiolitis. The patients recruited for this study were 6 to 24 months old and hospitalized with their first episode of acute RSV bronchiolitis (severe viral wheezing). Each participant was treated with 4-mg montelukast granules (RSV-MONT group) or a matching placebo (RSV-PLC group) for 3 months. This was the first placebo-controlled clinical trial of respiratory medicine in Korean children.

The primary endpoint was serum EDN level measurement, while the secondary endpoint was cumulative recurrent wheezing episodes for 12 months. Comparisons were made with normal controls. The baseline serum EDN levels in infants with acute RSV were significantly higher than in age-matched control subjects $(n=50)$; interestingly, this was the first report on elevated serum EDN levels in acute and post-RSV bronchiolitis. At the end of the 3-month treatment period, the RSV-PLC group $(\mathrm{n}=71)$ had significantly increased EDN levels $(P<0.0001)$, while the RSV-MONT group $(\mathrm{n}=79)$ had significantly decreased EDN levels $(P<0.01)$ compared to baseline. This treatment effect lasted for the entire 12-month study period. Furthermore, the RSV-PLC group had significantly higher serum EDN levels than the RSV-MONT $(P<0.001)$ and control $(P<0.001)$ groups 
throughout the study period ${ }^{19)}$.

An interesting finding was that 3-month serum EDN levels correlated significantly with the total number of wheezing episodes at 12 months in both the RSV-PLC $(\mathrm{r}=0.720, P<0.0001)$ and RSV-MONT ( $\mathrm{r}=0.531, P<0.001)$ groups. With $53 \mathrm{ng} / \mathrm{mL}$ (median+2 standard deviations) as the cutoff point for an elevated EDN level, the positive predictive value of the 3-month serum EDN level for the cumulative number of recurrent wheezing episodes at the 12-month point was 57\%; the negative predictive value was $76 \%$; the sensitivity was $72 \%$; and the specificity was $62 \%{ }^{19)}$.

It was concluded that elevated serum EDN levels were most likely caused by an increase in eosinophil degranulation during infantile wheezing ${ }^{19)}$. However, in the absence of premorbid levels, the idea that a preexisting allergy contributed to the persistence of high EDN levels could not be excluded. Viral infection in sensitized individuals (e.g., those with allergies or atopic heredity) may induce the release of eosinophil chemoattractants such as eotaxin from airway epithelial cells, resulting in increased EDN levels ${ }^{20}$. Genetic heterogeneity (e.g., Korean vs. Caucasian children) may have also contributed to high serum EDN levels ${ }^{21)}$.

The major conclusions from this study were that LTRA treatment reduced eosinophil degranulation during postviral wheezing and that LTRA treatment was associated with a decrease in wheezing episodes postbronchiolitis. These findings suggest that serum EDN levels are a useful biomarker for diagnosing and monitoring postviral wheezing. Furthermore, a high serum EDN levels at 3 months could be a predictive biomarker for recurrent wheezing/asthma development ${ }^{19)}$.

\section{EDN in childhood asthma: correlation with disease severity}

Total eosinophil counts (TECs) and ECP levels have been proposed as markers of disease activity. However, the usefulness of EDN as a marker in pediatric asthma has not been established yet. The objective of this study was to compare TECs and serum ECP and EDN levels with asthma symptom severity in young children.

In total, 43 young children with asthma (mean age, 2.9 years; range, 1.4 to 5.0 years) were evaluated during both the acute and stable phases of disease. Asthma severity was measured using a previously published symptom-scoring technique (mild, 1 to 3; moderate, 4 to 6; severe, 6 to 9) ${ }^{22}$. Nineteen age-matched controls were used for comparison.

TECs and serum EDN and ECP levels were measured in the acute and stable phases of disease as well as in controls. Only serum EDN levels were different between acute and SA phases $(P<0.0001)$ and between SA and controls $(P<0.0001)$. The patients were divided into 3 groups based on asthma severity, and only serum EDN levels were significantly different among all 3 groups (mild vs. moderate, $P<0.01$; moderate vs. severe, $P<0.0001$; mild vs. severe, $P<0.0001)$. Serum EDN levels showed the greatest correlation with symptom severity score (Rs=0.850, $P<0.0001)$ during the acute phase when compared to serum ECP levels and TECs. Serum EDN levels also showed the greatest significant change $(P<0.0001)$ between disease phases (i.e., acute vs. stable) when compared to serum ECP levels $(P<0.01)$ and TEC levels $(P<0.01)^{23)}$.

It was concluded that serum EDN level is also a useful biomarker for asthma pathophysiology and may better reflect asthma severity than ECP level or TEC. With further study, serum EDN levels may provide an objective, repeatable, and reproducible measure of the eosinophil's secretory activity and may be a more useful monitoring tool than serum ECP levels and TECs ${ }^{23)}$.

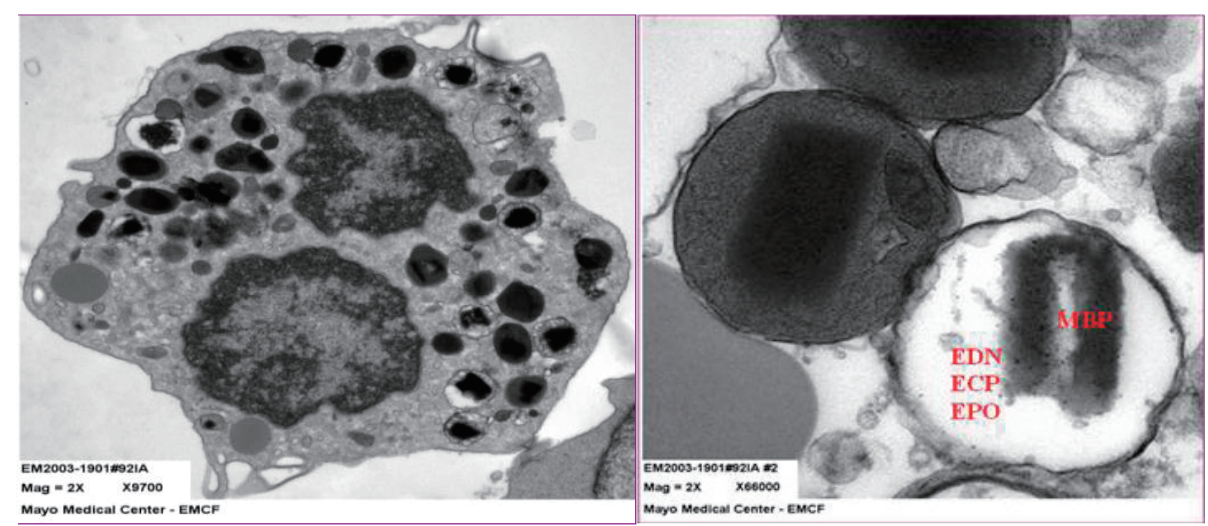

Fig. 1. (A) The transmission electron microscopic (EM) findings for intact sputum eosinophils. Characteristic dense core granules are seen. (B) Intact dense core granules and electron-lucent (degranulated) granules. EDN, eosinophil-derived neurotoxin; ECP, eosinophil cationic protein; EPO, eosinophil peroxidase; MBP, major basic protein. 


\section{EDN in other specimen types}

EDN levels can also be measured in induced sputum (IS), bronchoalveolar lavage fluid (BALF), and nasal lavage fluid (NLF). Each specimen type has its advantages and disadvantages compared to serum. For example, serum is always available and easy to obtain compared to other specimen types.

\section{IS EDN in children with SA}

IS was obtained from $30 \mathrm{SA}, 21 \mathrm{AR}$, and 22 nonatopic healthy control (HC) children. All children were 7 to 15 years of age. Sputum EDN levels in the SA group were significantly higher than those in the AR $(P<0.01)$ and HC groups $(P<$ $0.0001)$. Sputum eotaxin correlated significantly with sputum EDN, ECP, and eosinophil percentage. However, sputum interleukin-5 correlated only with sputum EDN levels, not sputum ECP levels or sputum eosinophil percentage ${ }^{24)}$. In conclusion, sputum EDN levels may aid differential diagnosis of asthma and AR.

\section{IS EDN in asthma and chronic cough (adult study)}

The objective of this study was to investigate whether eosinophil degranulation is a distinctive feature of asthma and whether it can be used for distinguishing between chronic cough patients with asthma and those without asthma. Thirtyseven patients with a chronic cough for $>1$ month and 9 normal individuals (controls) were enrolled.

The sputum eosinophil percentage was increased in the asthma $(P<0.001)$ and nonasthma $(P<0.05)$ groups compared with the control group. Sputum EDN levels were increased in the asthma group compared with the nonasthma $(P<0.05)$ and control $(P<0.05)$ groups. However, EDN levels were not increased in the nonasthma group compared with the control group. These findings suggest that eosinophil degranulation is more important than eosinophilia in identifying asthma ${ }^{25}$.

\section{Individual clinical cases monitoring with EDN}

EDN can be used to monitor asthma treatment. For example, the treatment efficacy of LTRA is demonstrated by a reduction in EDN levels (i.e., a reduction in eosinophil degranulation) from elevated values to normal values (13 to $45 \mathrm{ng} / \mathrm{mL})^{19)}$.

EDN levels also reflect the natural course of asthma. Without treatment, EDN concentrations increase from values within the normal range to significantly elevated levels.

\section{Conclusions}

As a monitoring tool, EDN has shown good results in children with asthma, as well as other allergic diseases. EDN can also be used in adult patients and in multiple specimen types (e.g., serum, sputum, BALF, and NLF). These results are repeatable and reproducible. In conclusion, serum EDN can be used as a novel biomarker for the diagnosis, treatment, and monitoring of asthma/allergic disease.

\section{Acknowledgment}

This study was supported by the 2012 Inje University Research Grant.

\section{References}

1. Hogan SP, Rosenberg HF, Moqbel R, Phipps S, Foster PS, Lacy P, et al. Eosinophils: biological properties and role in health and disease. Clin Exp Allergy 2008;38:709-50.

2. The Global Initiative for Asthma. GINA report, global strategy for asthma management and prevention. Updated Dec. 2011 [Internet]. [place unknown]: The Global Initiative For Asthma; c2011. [cited 2011 Jun 21]. Available from: http://www.ginasthma.org/guidelinesgina-report-global-strategy-for-asthma.html.

3. British Thoracic Society. 2008 British guideline on the management of asthma: a national clinical guideline. Updated Jun. 2009 [Internet]. London: British Thoracic Society; c2011 [cited 2011 Jun 21]. Available from: http://www.brit-thoracic.org.uk/guidelines/ asthma-guidelines.aspx.

4. Crimi E, Spanevello A, Neri M, Ind PW, Rossi GA, Brusasco V. Dissociation between airway inflammation and airway hyperresponsiveness in allergic asthma. Am J Respir Crit Care Med 1998;157:4-9.

5. Parameswaran K, Pizzichini E, Pizzichini MM, Hussack P, Efthimiadis A, Hargreave FE. Clinical judgement of airway inflammation versus sputum cell counts in patients with asthma. Eur Respir J 2000;15:486-90.

6. Pizzichini MM, Pizzichini E, Clelland L, Efthimiadis A, Pavord I, Dolovich J, et al. Prednisone-dependent asthma: inflammatory indices in induced sputum. Eur Respir J 1999;13:15-21.

7. Jatakanon A, Lim S, Barnes PJ. Changes in sputum eosinophils predict loss of asthma control. Am J Respir Crit Care Med 2000;161:64-72.

8. Venge P. Monitoring the allergic inflammation. Allergy 2004;59: 26-32.

9. Gleich GJ, Adolphson CR. The eosinophil and bronchial asthma: evidence for a critical role of eosinophils in pathophysiology. In: Sanderson CJ, editor., Interleukin-5: from molecule to drug target for asthma (lung biology in health disease). New York: Marcel Dekker, 1999:1-37.

10. Kim KW, Lee KE, Kim ES, Song TW, Sohn MH, Kim KE. Serum eosinophil-derived neurotoxin (EDN) in diagnosis and evaluation of severity and bronchial hyperresponsiveness in childhood asthma. Lung 2007;185:97-103.

11. Taniuchi S, Chihara J, Kojima T, Yamamoto A, Sasai M, Kobayashi Y. Serum eosinophil derived neurotoxin may reflect more strongly disease severity in childhood atopic dermatitis than eosinophil cationic protein. J Dermatol Sci 2001;26:79-82.

12. Wilson NM, Bridge P, Spanevello A, Silverman M. Induced sputum in children: feasibility, repeatability, and relation of findings to asthma severity. Thorax 2000;55:768-74. 
13. Wojnarowski C, Roithner B, Koller DY, Halmerbauer G, Gartner C, Tauber E, et al. Lack of relationship between eosinophil cationic protein and eosinophil protein $\mathrm{X}$ in nasal lavage and urine and the severity of childhood asthma in a 6-month follow-up study. Clin Exp Allergy 1999;29: 926-32.

14. Gleich GJ, Adolphson CR. The eosinophilic leukocyte: structure and function. Adv Immunol 1986;39:177-253.

15. Venge P, Bystrom J, Carlson M, Hakansson L, Karawacjzyk M, Peterson C, et al. Eosinophil cationic protein (ECP): molecular and biological properties and the use of ECP as a marker of eosinophil activation in disease. Clin Exp Allergy 1999;29:1172-86.

16. Goto T, Morioka J, Inamura H, Yano M, Kodaira K, Igarashi Y, et al. Urinary eosinophil-derived neurotoxin concentrations in patients with atopic dermatitis: a useful clinical marker for disease activity. Allergol Int 2007;56:433-8.

17. Koller DY, Halmerbauer G, Frischer T, Roithner B. Assessment of eosinophil granule proteins in various body fluids: is there a relation to clinical variables in childhood asthma? Clin Exp Allergy 1999;29:786-93.

18. Harrison AM, Bonville CA, Rosenberg HF, Domachowske JB. Respiratory syncytical virus-induced chemokine expression in the lower airways: eosinophil recruitment and degranulation. Am J Respir Crit Care Med 1999;159:1918-24.

19. Kim CK, Choi J, Kim HB, Callaway Z, Shin BM, Kim JT, et al. A randomized intervention of montelukast for post-bronchiolitis: effect on eosinophil degranulation. J Pediatr 2010;156:749-54.

20. Papadopoulos NG, Papi A, Meyer J, Stanciu LA, Salvi S, Holgate ST, et al. Rhinovirus infection up-regulates eotaxin and eotaxin-2 expression in bronchial epithelial cells. Clin Exp Allergy 2001;31: 1060-6.

21. Choi EH, Lee HJ, Yoo T, Chanock SJ. A common haplotype of interleukin-4 gene IL4 is associated with severe respiratory syncytial virus disease in Korean children. J Infect Dis 2002;186: 1207-11.

22. Parkin PC, Macarthur C, Saunders NR, Diamond SA, Winders PM. Development of a clinical asthma score for use in hospitalized children between 1 and 5 years of age. J Clin Epidemiol 1996;49: 821-5.

23. Kim CK, Callaway Z, Fletcher R, Koh YY. Eosinophil-derived neurotoxin in childhood asthma: correlation with disease severity. J Asthma 2010;47:568-73.

24. Kim CK, Kita H, Callaway Z, Kim HB, Choi J, Fujisawa T, et al. The roles of a Th2 cytokine and CC chemokine in children with stable asthma: potential implication in eosinophil degranulation. Pediatr Allergy Immunol 2010;21(4 Pt 2):e697-704.

25. Kim CK, Callaway Z, Kim DW, Kita H. Eosinophil degranulation is more important than eosinophilia in identifying asthma in chronic cough. J Asthma 2011;48:994-1000. 